\title{
On the Assessment of Acoustic Scattering and Shielding by Time Domain Boundary Integral Equation Solutions
}

\author{
Fang Q. $\mathrm{Hu}^{*}$ and Michelle E. Pizzo ${ }^{\dagger}$ \\ Old Dominion University, Norfolk, Virginia 23529 \\ Douglas M. Nark ${ }^{\ddagger}$ \\ NASA Langley Research Center, Hampton, Virginia 23681
}

\begin{abstract}
Based on the time domain boundary integral equation formulation of the linear convective wave equation, a computational tool dubbed Time Domain Fast Acoustic Scattering Toolkit (TD-FAST) has recently been under development. The time domain approach has a distinct advantage that the solutions at all frequencies are obtained in a single computation. In this paper, the formulation of the integral equation, as well as its stabilization by the Burton-Miller type reformulation, is extended to cases of a constant mean flow in an arbitrary direction. In addition, a "Source Surface" is also introduced in the formulation that can be employed to encapsulate regions of noise sources and to facilitate coupling with CFD simulations. This is particularly useful for applications where the noise sources are not easily described by analytical source terms. Numerical examples are presented to assess the accuracy of the formulation, including a computation of noise shielding by a thin barrier motivated by recent Historical Baseline F31A31 open rotor noise shielding experiments. Furthermore, spatial resolution requirements of the time domain boundary element method are also assessed using point per wavelength metrics. It is found that, using only constant basis functions and high-order quadrature for surface integration, relative errors of less than $2 \%$ may be obtained when the surface spatial resolution is 5 points-per-wavelength (PPW) or 25 points-per-wavelengthsquared $\left(\mathrm{PPW}^{2}\right)$.
\end{abstract}

\section{Introduction}

In developing the next generation quieter aircraft, there is a critical need to accurately and efficiently predict the acoustic scattering and shielding by the aircraft body, rigid as well as lined, from given noise sources. Computation of acoustic wave propagation and interaction with solid or treated surfaces is also important for the prediction of airframe noise. Recently, a computational tool dubbed Time Domain Fast Acoustic Scattering Toolkit (TD-FAST) has been under development. ${ }^{10,11}$ It is based on the time domain boundary integral equation formulation of the linear convective wave equation. The time domain approach has a distinct advantage that the solutions at all frequencies are obtained in a single computation. The boundary element method used for the solution of the

${ }^{*}$ Professor, Department of Mathematics and Statistics, Associate Fellow AIAA

${ }^{\dagger}$ Graduate Student, Department of Mathematics and Statistics, Member AIAA

${ }^{\ddagger}$ Senior Research Scientist, Research Directorate, Structural Acoustics Branch, Associate Fellow AIAA 
boundary integral equation eliminates the need of a volume mesh that could be prohibitively large for simulation of waves at high frequencies. To increase computational efficiency, numerical solution of the boundary integral equation is accelerated by the use of GPU computing ${ }^{10}$ and the multi-level Time-Domain Propagation and Distribution (TDPD) algorithm, ${ }^{10}$ which is based on the Cartesian Non-uniform Grid Time Domain algorithm (CNGTDA). ${ }^{2,3,15}$

In this paper, formulation of the time domain integral equation will be extended to cases of a constant mean flow in a general direction. The formulation presented in ref. ${ }^{10}$ was restricted to a mean flow aligned with the $x$-axis. The new time domain integral equation presented in the current paper, as well as its stabilization by the Burton-Miller reformulation, will be given and validated numerically where the mean flow can be in an arbitrary direction. In addition, to facilitate the coupling of TD-FAST with time domain CFD simulations, a "Source Surface" is introduced in the new formulation. Such a source surface can be employed to encapsulate a region of noise sources that may be computed independently. This is particularly useful for applications where the noise sources are not easily described by analytical source terms.

Following the formulation, an application of TD-FAST to the scattering and shielding of sound by a thin barrier is presented. This example is motivated by the recent experimental results of the F31A31 historical baseline open rotor noise shielding wind tunnel tests. ${ }^{18}$ However, in these initial predictions, the open rotor is modeled as a point source to establish the problem geometry and ultimately illustrate the effect of the source model on the scattered field. The source surface approach is also used and the prediction methodology validated through comparisons with a known analytical solution for sound diffraction. In addition, the accuracy and resolution of the boundary element approach is further assessed by analyzing the computational errors using points-per-wavelength metrics. Studies on spatial resolution can provide a practical guidance on required mesh density for the range of frequencies to be included in the time domain computation.

The rest of the paper is organized as follows. In Section 2, formulation of the time domain boundary integral equation for a constant mean flow in an arbitrary direction and its reformulation by the Burton-Miller approach are presented. The new formulation also includes the possibility of source surfaces. In Section 3, the validity of the time domain integral equation is verified by substituting an analytical solution of the convective wave equation into the integral formulation. In Section 4 , sound scattering and shielding by a thin barrier are computed in the time domain using TDFAST and the converted frequency domain results are compared with the analytical solution of the scattered sound of a semi-infinite plate. In Section 5, points-per-wavelength requirements on the accuracy of the boundary element method are discussed. Finally, concluding remarks are contained in Section 6.

\section{Formulation of the time domain boundary integral equation}

\section{A. Time domain integral relations}

We consider the convective wave equation for a constant mean flow $\boldsymbol{U}$ written as

$$
\left(\frac{\partial}{\partial t}+\boldsymbol{U} \cdot \nabla\right)^{2} p-c^{2} \nabla^{2} p=q(\boldsymbol{r}, t)
$$

with homogeneous initial condition

$$
p(\boldsymbol{r}, 0)=\frac{\partial p}{\partial t}(\boldsymbol{r}, 0)=0, \quad t=0
$$


Here $q(\boldsymbol{r}, t)$ represents known source terms. In addition, we also assume that there could be a "Source Surface" on which both $p(\boldsymbol{r}, t)$ and its normal derivative $\frac{\partial p}{\partial n}(\boldsymbol{r}, t)$ are specified:

$$
p(\boldsymbol{r}, t)=f(\boldsymbol{r}, t), \quad \frac{\partial p}{\partial n}(\boldsymbol{r}, t)=g(\boldsymbol{r}, t), \quad \boldsymbol{r} \in S_{0}
$$

The source surface is introduced to conveniently encapsulate source regions and to facilitate the coupling with CFD simulations. Therefore, the sources for a wave propagation and scattering problem can be described as source terms on the right hand side of the wave equation (1), a given solution on a specified surface (3), or both. The source surface will be denoted by $S_{0}$ and the surface of the scattering body will be denoted by $S_{b}$, as illustrated in Figure 1. On an acoustically hard surface, we will have the following boundary condition:

$$
\frac{\partial p}{\partial n}(\boldsymbol{r}, t)=0, \quad \boldsymbol{r} \in S_{b}
$$

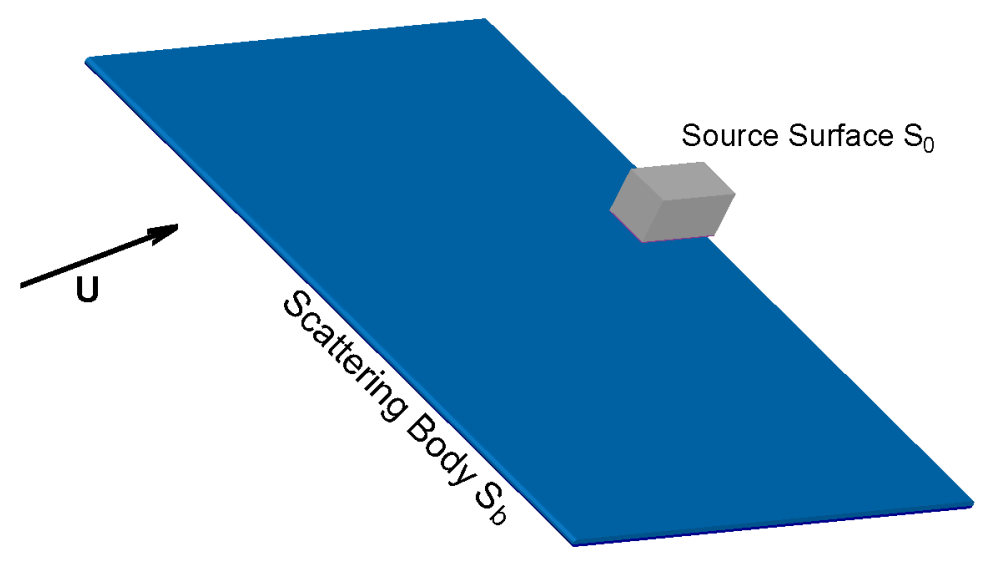

Figure 1. A schematic diagram showing the scattering body $S_{b}$ and source surface $S_{0}$.

The partial differential equation (1), together with the initial and boundary conditions, can be converted into a time-domain boundary integral equation. For the special case of a mean flow $\boldsymbol{U}$ that is in the direction of the x-axis, a derivation of the integral equation, through the free space adjoint Green's function, has been given in detail in ref. ${ }^{10}$ In what follows, we present the time domain boundary integral equation for a constant mean flow in an arbitrary direction and for the inclusion of the source surface.

The free space Green's function of the adjoint equation is well-known $6,9,17,21$ and can be written as

$$
\tilde{G}\left(\boldsymbol{r}, t ; \boldsymbol{r}^{\prime}, t^{\prime}\right)=\frac{G_{0}}{4 \pi c^{2}} \delta\left(t^{\prime}-t+\boldsymbol{\beta} \cdot\left(\boldsymbol{r}^{\prime}-\boldsymbol{r}\right)-\frac{\bar{R}}{c \alpha^{2}}\right)
$$

where

$$
G_{0}=\frac{1}{\bar{R}\left(\boldsymbol{r}, \boldsymbol{r}^{\prime}\right)}, \text { and } \bar{R}\left(\boldsymbol{r}, \boldsymbol{r}^{\prime}\right)=\sqrt{\left[\boldsymbol{M} \cdot\left(\boldsymbol{r}-\boldsymbol{r}^{\prime}\right)\right]^{2}+\alpha^{2}\left|\boldsymbol{r}-\boldsymbol{r}^{\prime}\right|^{2}}
$$

in which 


$$
\boldsymbol{M}=\frac{\boldsymbol{U}}{c}, \quad \alpha=\sqrt{1-(U / c)^{2}}=\sqrt{1-M^{2}}, \quad \boldsymbol{\beta}=\frac{\boldsymbol{U}}{c^{2}-U^{2}}=\frac{\boldsymbol{U}}{c^{2} \alpha^{2}}=\frac{\boldsymbol{M}}{c \alpha^{2}}, \quad U=|\boldsymbol{U}|, \quad M=|\boldsymbol{M}|
$$

with $\boldsymbol{U}$ being the constant velocity vector and $c$ the speed of sound. The arguments for $\bar{R}\left(\boldsymbol{r}, \boldsymbol{r}^{\prime}\right)$ will be omitted when there is no misunderstanding for doing so.

As shown by $\mathrm{Hu},{ }^{10}$ the time domain boundary integral equation for acoustic scattering is the following,

$$
\begin{gathered}
4 \pi C_{s} p\left(\boldsymbol{r}^{\prime}, t^{\prime}\right)=\frac{1}{c^{2}} \int_{V} \frac{1}{\bar{R}} q\left(\boldsymbol{r}^{\prime}, t_{R}^{\prime}\right) d \boldsymbol{r}+\int_{S}\left[\left(1-M_{n}^{2}\right) G_{0} \frac{\partial p}{\partial n}\left(\boldsymbol{r}_{s}, t_{R}^{\prime}\right)-\frac{\partial G_{0}}{\partial \bar{n}}\left(p\left(\boldsymbol{r}_{s}, t_{R}^{\prime}\right)+\frac{\bar{R}}{c \alpha^{2}} \frac{\partial p}{\partial t}\left(\boldsymbol{r}_{s}, t_{R}^{\prime}\right)\right)\right. \\
\left.-M_{n} G_{0}\left(\boldsymbol{M}_{T} \cdot \nabla p\left(\boldsymbol{r}_{s}, t_{R}^{\prime}\right)+\frac{1}{c} \frac{\partial p}{\partial t}\left(\boldsymbol{r}_{s}, t_{R}^{\prime}\right)\right)\right] d \boldsymbol{r}_{s}
\end{gathered}
$$

where $S$ denotes the surfaces of both the scattering body and the possible source surface $S_{0}$, i.e.,

$$
S=S_{b} \cup S_{0}
$$

and $C_{s}$ denotes a constant whose value is as follows,

$$
C_{s}= \begin{cases}1 & \boldsymbol{r}^{\prime} \text { in the exterior of } S \\ \frac{1}{2} & \boldsymbol{r}^{\prime} \text { on } S \text { (smooth points) } \\ 0 & \boldsymbol{r}^{\prime} \text { in the interior of } S\end{cases}
$$

In (8), $M_{n}$ and $\boldsymbol{M}_{T}$ denote, respectively, the normal and tangential components of the mean flow on surface $\mathrm{S}$ :

$$
M_{n}=\boldsymbol{M} \cdot \boldsymbol{n}, \quad \boldsymbol{M}_{T}=\boldsymbol{M}-M_{n} \boldsymbol{n}
$$

The retarded time $t_{R}^{\prime}$ appearing in (8) is defined as

$$
t_{R}^{\prime}=t^{\prime}+\boldsymbol{\beta} \cdot\left(\boldsymbol{r}^{\prime}-\boldsymbol{r}\right)-\frac{\bar{R}}{c \alpha^{2}}
$$

Also, denoted by an overbar, $\bar{n}$ in (8) is a modified normal ${ }^{10}$ and defined as

$$
\overline{\boldsymbol{n}}=\boldsymbol{n}-M_{n} \boldsymbol{M}, \text { and } \frac{\partial}{\partial \bar{n}}=\frac{\partial}{\partial n}-M_{n}(\boldsymbol{M} \cdot \nabla)=\left(\boldsymbol{n}-M_{n} \boldsymbol{M}\right) \cdot \nabla
$$

The modified normal derivatives are to be computed as follows,

$$
\frac{\partial \bar{R}}{\partial \bar{n}}=\frac{\partial \bar{R}}{\partial n}-M_{n}(\boldsymbol{M} \cdot \nabla \bar{R})=\alpha^{2} \frac{\boldsymbol{n} \cdot\left(\boldsymbol{r}-\boldsymbol{r}^{\prime}\right)}{\bar{R}}
$$

and

$$
\frac{\partial G_{0}}{\partial \bar{n}}=-\frac{1}{\bar{R}^{2}} \frac{\partial \bar{R}}{\partial \bar{n}}=-\alpha^{2} \frac{\boldsymbol{n} \cdot\left(\boldsymbol{r}-\boldsymbol{r}^{\prime}\right)}{\bar{R}^{3}}
$$


On the source surface $S_{0}$, both $p(\boldsymbol{r}, t)$ and $\frac{\partial p}{\partial n}(\boldsymbol{r}, t)$ are known as specified in (3). On the scattering surface $S_{b}$, either $p(\boldsymbol{r}, t)$ or $\frac{\partial p}{\partial n}(\boldsymbol{r}, t)$, or a combination of the two, is assumed to be known. For instance, if $S_{b}$ is a solid surface, $\frac{\partial p}{\partial n}$ is assumed to be zero and $p$ on $S_{b}$ is to be found by solving the integral equation generated by letting $\boldsymbol{r}^{\prime}=\boldsymbol{r}_{s}^{\prime}$ in (8). ${ }^{10}$

\section{B. Stable time domain integral equation by Burton-Miller type reformulation}

Direct numerical solution of the boundary integral equation formed by (8), however, can lead to numerical instabilities. ${ }^{7,16,20,21}$ For the time domain boundary integral equations, it has been found that a Burton-Miller type reformulation can be effective in eliminating the instability. 5, 7,8,12 For the convective wave equation, it was proposed ${ }^{10}$ that the Burton-Miller reformulation be formed by a linear combination of the time and modified normal derivatives of the boundary integral equation. A theoretical justification was also presented ${ }^{10}$ where it was shown that under the proposed reformulation of the Burton-Miller type, there will be no nontrivial solution for the interior domain. For a general mean flow $\boldsymbol{U}$ considered here, by taking a combination of the time and modified normal derivatives of the form

$$
a \frac{\partial}{\partial t^{\prime}}+b c \frac{\partial}{\partial \bar{n}^{\prime}}
$$

where $a$ and $b$ are constants and $c$ is the speed of sound, the Burton-Miller reformulation leads to the following,

$$
\begin{gathered}
4 \pi a C_{s} \frac{\partial p\left(\boldsymbol{r}_{s}^{\prime}, t^{\prime}\right)}{\partial t}+4 \pi b c \frac{\partial C_{s}}{\partial \bar{n}^{\prime}} p\left(\boldsymbol{r}_{s}^{\prime}, t^{\prime}\right)+4 \pi b c C_{s} \frac{\partial p\left(\boldsymbol{r}_{s}^{\prime}, t^{\prime}\right)}{\partial \bar{n}^{\prime}}=a \frac{1}{c^{2}} \int_{V} \frac{1}{\bar{R}} \frac{\partial q}{\partial t}\left(\boldsymbol{r}_{s}^{\prime}, t_{R}^{\prime}\right) d \boldsymbol{r}+\frac{b}{c} \frac{\partial}{\partial \bar{n}^{\prime}} \int_{V} \frac{1}{\bar{R}} q\left(\boldsymbol{r}_{s}^{\prime}, t_{R}^{\prime}\right) d \boldsymbol{r} \\
+a \int_{S}\left[\left(1-M_{n}^{2}\right) G_{0} \frac{\partial p_{n}}{\partial t}\left(\boldsymbol{r}_{s}, t_{R}^{\prime}\right)-\frac{\partial G_{0}}{\partial \bar{n}}\left(\frac{\partial p}{\partial t}\left(\boldsymbol{r}_{s}, t_{R}^{\prime}\right)+\frac{\bar{R}}{c \alpha^{2}} \frac{\partial^{2} p}{\partial t^{2}}\left(\boldsymbol{r}_{s}, t_{R}^{\prime}\right)\right)-M_{n} G_{0}\left(\boldsymbol{M}_{T} \cdot \nabla \frac{\partial p}{\partial t}\left(\boldsymbol{r}_{s}, t_{R}^{\prime}\right)+\frac{1}{c} \frac{\partial^{2} p}{\partial t^{2}}\left(\boldsymbol{r}_{s}, t_{R}^{\prime}\right)\right)\right] d \boldsymbol{r}_{s} \\
+b c \int_{S}\left[\left(1-M_{n}^{2}\right) \frac{\partial G_{0}}{\partial \bar{n}^{\prime}} p_{n}\left(\boldsymbol{r}_{s}, t_{R}^{\prime}\right)+\left(1-M_{n}^{2}\right) G_{0} \frac{\partial p_{n}}{\partial \bar{n}^{\prime}}\left(\boldsymbol{r}_{s}, t_{R}^{\prime}\right)\right] d \boldsymbol{r} \\
-b c \int_{S}\left[\frac{\partial^{2} G_{0}}{\partial \bar{n}^{\prime} \partial \bar{n}}\left(p\left(\boldsymbol{r}_{s}, t_{R}^{\prime}\right)+\frac{\bar{R}}{c \alpha^{2}} \frac{\partial p}{\partial t}\left(\boldsymbol{r}_{s}, t_{R}^{\prime}\right)\right)+M_{n} \frac{\partial G_{0}}{\partial \bar{n}^{\prime}}\left(\boldsymbol{M}_{T} \cdot \nabla p\left(\boldsymbol{r}_{s}, t_{R}^{\prime}\right)+\frac{1}{c} \frac{\partial p}{\partial t}\left(\boldsymbol{r}_{s}, t_{R}^{\prime}\right)\right)\right] d \boldsymbol{r}_{s} \\
-\frac{b}{\alpha^{2}} \int_{S} \frac{\partial G_{0}}{\partial \bar{n}}\left[\left(\boldsymbol{M} \cdot \overline{\boldsymbol{n}}^{\prime}\right) \frac{\partial p}{\partial t}\left(\boldsymbol{r}_{s}, t_{R}^{\prime}\right)+\frac{\bar{R}}{c \alpha^{2}}\left(\boldsymbol{M} \cdot \overline{\boldsymbol{n}}^{\prime}-\frac{\partial \bar{R}}{\partial \bar{n}^{\prime}}\right) \frac{\partial^{2} p}{\partial t^{2}}\left(\boldsymbol{r}_{s}, t_{R}^{\prime}\right)\right] d \boldsymbol{r}_{s} \\
-\frac{b}{\alpha^{2}} \int_{S} M_{n} G_{0}\left(\boldsymbol{M} \cdot \overline{\boldsymbol{n}}^{\prime}-\frac{\partial \bar{R}}{\partial \bar{n}^{\prime}}\right)\left(\boldsymbol{M}_{T} \cdot \nabla \frac{\partial p}{\partial t}\left(\boldsymbol{r}_{s}, t_{R}^{\prime}\right)+\frac{1}{c} \frac{\partial^{2} p}{\partial t^{2}}\left(\boldsymbol{r}_{s}, t_{R}^{\prime}\right)\right) d \boldsymbol{r}_{s}
\end{gathered}
$$

We note that, unlike the previously presented derivation, ${ }^{10}$ the terms associated with the pressure normal derivative $\frac{\partial p}{\partial n}$ are retained due to the inclusion of the source surface. For brevity, we have used $p_{n}\left(\boldsymbol{r}_{s}, t\right)$ to denote the normal derivative of $p$ on the surface point $\boldsymbol{r}_{s}$, i.e., $p_{n}\left(\boldsymbol{r}_{s}, t\right) \equiv \frac{\partial p}{\partial n}\left(\boldsymbol{r}_{s}, t\right)$. Its modified normal derivative that appeared in (11) is to be computed as

$$
\frac{\partial p_{n}}{\partial \bar{n}^{\prime}}\left(\boldsymbol{r}_{s}, t_{R}^{\prime}\right)=\frac{\partial p_{n}}{\partial t}\left(\boldsymbol{r}_{s}, t_{R}^{\prime}\right) \frac{\partial t_{R}^{\prime}}{\partial \bar{n}^{\prime}}=\frac{\partial p_{n}}{\partial t}\left(\boldsymbol{r}_{s}, t_{R}^{\prime}\right)\left[\boldsymbol{\beta} \cdot \overline{\boldsymbol{n}}^{\prime}-\frac{1}{c \alpha^{2}} \frac{\partial \bar{R}}{\partial \bar{n}^{\prime}}\right]=\frac{1}{c \alpha^{2}} \frac{\partial p_{n}}{\partial t}\left(\boldsymbol{r}_{s}, t_{R}^{\prime}\right)\left[\boldsymbol{M} \cdot \overline{\boldsymbol{n}}^{\prime}-\frac{\partial \bar{R}}{\partial \bar{n}^{\prime}}\right]
$$

Also, for a mean flow in a general direction, the double modified normal derivative of the $G_{0}$ is to be computed as the following: 


$$
\frac{\partial^{2} G_{0}}{\partial \bar{n}^{\prime} \partial \bar{n}}=\frac{\partial}{\partial \bar{n}^{\prime}}\left[-\alpha^{2} \frac{\boldsymbol{n} \cdot\left(\boldsymbol{r}-\boldsymbol{r}^{\prime}\right)}{\bar{R}^{3}}\right]=\frac{\alpha^{2}}{\bar{R}^{3}}\left[\boldsymbol{n} \cdot \boldsymbol{n}^{\prime}-M_{n^{\prime}} M_{n}\right]+3 \alpha^{4} \frac{\left[\boldsymbol{n} \cdot\left(\boldsymbol{r}-\boldsymbol{r}^{\prime}\right)\right]\left[\boldsymbol{n}^{\prime} \cdot\left(\boldsymbol{r}^{\prime}-\boldsymbol{r}\right)\right]}{\bar{R}^{5}}
$$

To deal with the hyper-singularity brought about by the term $\frac{\partial^{2} G_{0}}{\partial \bar{n}^{\prime} \partial \bar{n}}$, we follow the method used by $\mathrm{Hu}^{10}$ and note that

$$
4 \pi \frac{\partial C_{s}}{\partial \bar{n}^{\prime}}=-\frac{\partial}{\partial \bar{n}^{\prime}} \int_{S} \frac{\partial G_{0}}{\partial \bar{n}} d \boldsymbol{r}_{s}=-\int_{S} \frac{\partial G_{0}^{2}}{\partial \bar{n}^{\prime} \partial \bar{n}} d \boldsymbol{r}_{s}
$$

By replacing $4 \pi \frac{\partial C_{s}}{\partial \bar{n}^{\prime}}$ in the second term on the left hand side of (11) with (12) and then moving it to the right hand side, the hyper-singularity is reduced and we get the following second version of the time domain boundary integral equation:

$$
\begin{gathered}
4 \pi a C_{s} \frac{\partial p\left(\boldsymbol{r}_{s}^{\prime}, t^{\prime}\right)}{\partial t}+4 \pi b c C_{s} \frac{\partial p\left(\boldsymbol{r}_{s}^{\prime}, t^{\prime}\right)}{\partial \bar{n}^{\prime}}=a \frac{1}{c^{2}} \int_{V} \frac{1}{\bar{R}} \frac{\partial q}{\partial t}\left(\boldsymbol{r}_{s}^{\prime}, t_{R}^{\prime}\right) d \boldsymbol{r}+\frac{b}{c} \frac{\partial}{\partial \bar{n}^{\prime}} \int_{V} \frac{1}{\bar{R}} \frac{\partial q}{\partial t}\left(\boldsymbol{r}_{s}^{\prime}, t_{R}^{\prime}\right) d \boldsymbol{r} \\
+a \int_{S}\left[\left(1-M_{n}^{2}\right) G_{0} \frac{\partial p_{n}}{\partial t}\left(\boldsymbol{r}_{s}, t_{R}^{\prime}\right)-\frac{\partial G_{0}}{\partial \bar{n}}\left(\frac{\partial p}{\partial t}\left(\boldsymbol{r}_{s}, t_{R}^{\prime}\right)+\frac{\bar{R}}{c \alpha^{2}} \frac{\partial^{2} p}{\partial t^{2}}\left(\boldsymbol{r}_{s}, t_{R}^{\prime}\right)\right)-M_{n} G_{0}\left(\boldsymbol{M}_{T} \cdot \nabla \frac{\partial p}{\partial t}\left(\boldsymbol{r}_{s}, t_{R}^{\prime}\right)+\frac{1}{c} \frac{\partial^{2} p}{\partial t^{2}}\left(\boldsymbol{r}_{s}, t_{R}^{\prime}\right)\right)\right] d \boldsymbol{r}_{s} \\
+b c \int_{S}\left[\left(1-M_{n}^{2}\right) \frac{\partial G_{0}}{\partial \bar{n}^{\prime}} p_{n}\left(\boldsymbol{r}_{s}, t_{R}^{\prime}\right)+\frac{1}{c \alpha^{2}}\left(1-M_{n}^{2}\right) G_{0}\left[\boldsymbol{M} \cdot \overline{\boldsymbol{n}}^{\prime}-\frac{\partial \bar{R}}{\partial \bar{n}^{\prime}}\right] \frac{\partial p_{n}}{\partial t}\left(\boldsymbol{r}_{s}, t_{R}^{\prime}\right)\right] d \boldsymbol{r} \\
-b c \int_{S}\left[\frac{\partial^{2} G_{0}}{\partial \bar{n}^{\prime} \partial \bar{n}}\left(p\left(\boldsymbol{r}_{s}, t_{R}^{\prime}\right)-p\left(\boldsymbol{r}_{s}^{\prime}, t^{\prime}\right)+\frac{\bar{R}}{c \alpha^{2}} \frac{\partial p}{\partial t}\left(\boldsymbol{r}_{s}, t_{R}^{\prime}\right)\right)+M_{n} \frac{\partial G_{0}}{\partial \bar{n}^{\prime}}\left(\boldsymbol{M}_{T} \cdot \nabla p\left(\boldsymbol{r}_{s}, t_{R}^{\prime}\right)+\frac{1}{c} \frac{\partial p}{\partial t}\left(\boldsymbol{r}_{s}, t_{R}^{\prime}\right)\right)\right] d \boldsymbol{r}_{s} \\
-\frac{b}{\alpha^{2}} \int_{S} \frac{\partial G_{0}}{\partial \bar{n}}\left[\left(\boldsymbol{M} \cdot \overline{\boldsymbol{n}}^{\prime}\right) \frac{\partial p}{\partial t}\left(\boldsymbol{r}_{s}, t_{R}^{\prime}\right)+\frac{\bar{R}}{c \alpha^{2}}\left(\boldsymbol{M} \cdot \overline{\boldsymbol{n}}^{\prime}-\frac{\partial \bar{R}}{\partial \bar{n}^{\prime}}\right) \frac{\partial^{2} p}{\partial t^{2}}\left(\boldsymbol{r}_{s}, t_{R}^{\prime}\right)\right] d \boldsymbol{r}_{s} \\
-\frac{b}{\alpha^{2}} \int_{S} M_{n} G_{0}\left(\boldsymbol{M} \cdot \overline{\boldsymbol{n}}^{\prime}-\frac{\partial \bar{R}}{\partial \bar{n}^{\prime}}\right)\left(\boldsymbol{M}_{T} \cdot \nabla \frac{\partial p}{\partial t}\left(\boldsymbol{r}_{s}, t_{R}^{\prime}\right)+\frac{1}{c} \frac{\partial^{2} p}{\partial t^{2}}\left(\boldsymbol{r}_{s}, t_{R}^{\prime}\right)\right) d \boldsymbol{r}_{s}
\end{gathered}
$$

With the reduction of the singularity, equation (13) is in a form that can be readily used for boundary element methods.

For numerical implementation of (13), we also note that

$$
\begin{gathered}
\frac{\partial p\left(\boldsymbol{r}_{s}^{\prime}, t^{\prime}\right)}{\partial \bar{n}^{\prime}}=\frac{\partial p\left(\boldsymbol{r}_{s}^{\prime}, t^{\prime}\right)}{\partial n^{\prime}}-M_{n^{\prime}} \boldsymbol{M} \cdot \nabla p\left(\boldsymbol{r}_{s}^{\prime}, t^{\prime}\right)=\left(1-M_{n^{\prime}}^{2}\right) p_{n}\left(\boldsymbol{r}_{s}^{\prime}, t^{\prime}\right)-M_{n^{\prime}} \boldsymbol{M}_{T^{\prime}} \cdot \nabla p\left(\boldsymbol{r}_{s}^{\prime}, t^{\prime}\right) \\
\boldsymbol{M} \cdot \bar{n}=\boldsymbol{M} \cdot\left(\boldsymbol{n}-M_{n} \boldsymbol{M}\right)=M_{n}-M_{n} M^{2}=M_{n} \alpha^{2}
\end{gathered}
$$

Furthermore, the tangential derivative terms such as $\boldsymbol{M}_{T} \cdot \nabla p\left(\boldsymbol{r}_{s}, t_{R}^{\prime}\right)$ can be found through the spatial derivatives of the surface basis functions as detailed previously. ${ }^{10}$

\section{Validation of time domain integral relation}

To assess the validity of the time domain integral relation presented in (8) for a constant mean flow in an arbitrary direction, we check the equation with a known exact solution of the convective wave equation. The exact solution for the acoustic pressure of a point source will be used for this validation. The pressure field of a point source located in $\boldsymbol{r}_{0}$ in a uniform mean flow $\boldsymbol{U}$ is

$$
p(\boldsymbol{r}, t)=\frac{1}{4 \pi c^{2} \bar{R}\left(\boldsymbol{r}, \boldsymbol{r}_{0}\right)} \psi\left(t+\boldsymbol{\beta} \cdot\left(\boldsymbol{r}-\boldsymbol{r}_{0}\right)-\frac{\bar{R}\left(\boldsymbol{r}, \boldsymbol{r}_{0}\right)}{c \alpha^{2}}\right)
$$


where $\psi(t)$ is a given time function for the source point. Here, a simple Gaussian function will be used,

$$
\psi(t)=e^{-\sigma(t / m \Delta t)^{2}}
$$

with $m=6$ and $\sigma=1.42$. For these parameters, the spectrum of the $\psi(t)$, also a Gaussian, reduces to a value that is 1000 th of its peak at a frequency of $1 / 6 \Delta t$.

We consider a source surface formed by a cubic box centered at $(0,0,0)$. The length of the box on each side is unity. Thus, the dimension of the source box is $[-0.5,0.5] \times[-0.5,0.5] \times[-0.5,0.5]$. The location of the point source is chosen to be inside the box at $r_{0}=(-0.25,0,0)$, so the analytical solution (14) satisfies the convective wave equation for the entire domain exterior of the box and hence the integral equation (8) with $q=0$. By prescribing the point source solution (14) on the surface of the cubic box, the integral equation (8) should reproduce the analytic solution for points outside, as well as, on the surface of the cubic box.
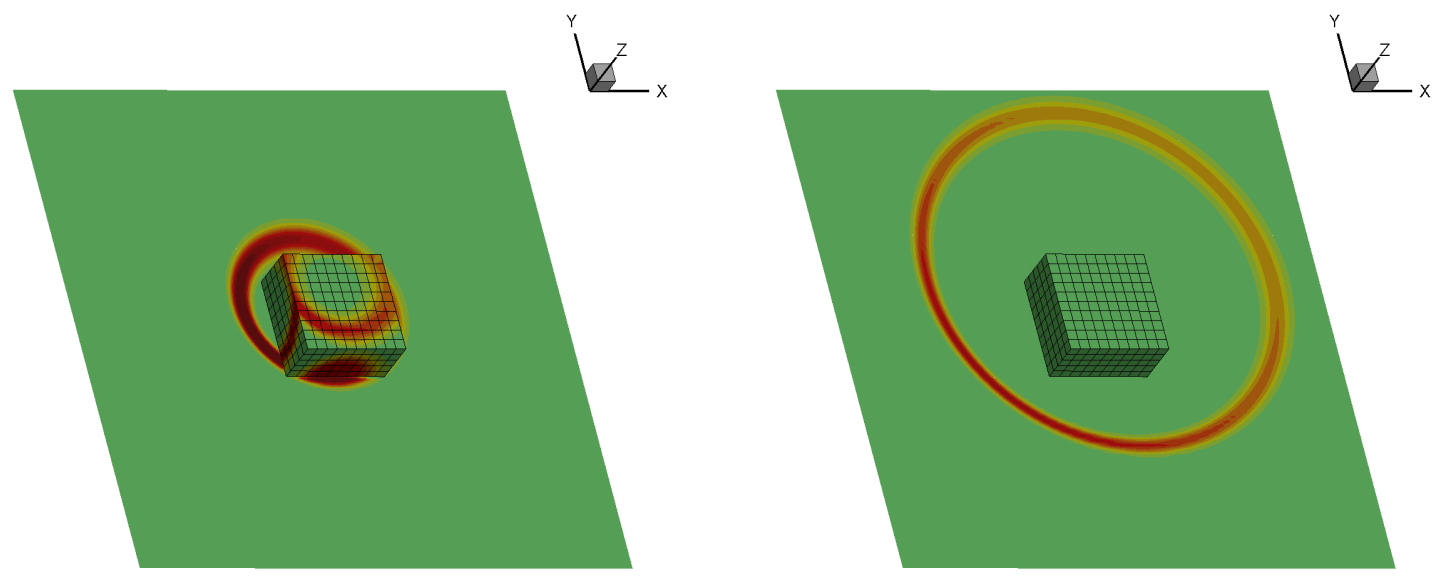

Figure 2. Instantaneous contours plots of pressure computed by (8) at two selected instances. Solutions for $p$ and $p_{n}$ are given on the cubic source surface and values on the field plane are computed by $(8)$.

In Figure 2, instantaneous pressure contours computed by (8) are shown, for a mean flow $\boldsymbol{M}=$ $(0.3,0.3,0)$. Each face of the source surface is discretized by $10 \times 10$ elements. The solution at a surface point $\boldsymbol{r}_{s}$ is discretized using spatial and temporal basis functions as

$$
p\left(\boldsymbol{r}_{s}, t\right)=\sum_{n=0}^{N_{t}} \sum_{i=1}^{N} u_{i}^{n} \phi_{i}\left(\boldsymbol{r}_{s}\right) \Psi\left(t-t_{n}\right)
$$

where $N$ is the total number of surface elements and $N_{t}$ is the total number of time steps. For the results shown here, second-order surface basis functions are used for $\phi_{i}\left(\boldsymbol{r}_{s}\right)$. $\Psi\left(t-t_{n}\right)$ denotes the temporal basis function for time node $t_{n}=n \Delta t$. The time step used for the current example is $\Delta t=0.02$. Further details for the time basis function can be found in $\mathrm{Hu} .{ }^{11}$

For a comparison between the numerical and analytical solutions, the values of pressure along $y=-1$ are plotted in Figure 3 at two chosen instances, $t=1.68$ and $t=2.48$. Excellent agreements are seen, which validates the integral equation (8) formulated for a constant mean flow in a general 
direction. The time history of pressure at two coordinates, $(-2,-1,0)$ and $(0.75,-1,0)$, are also shown in Figure 4, that again matches well with the exact value.

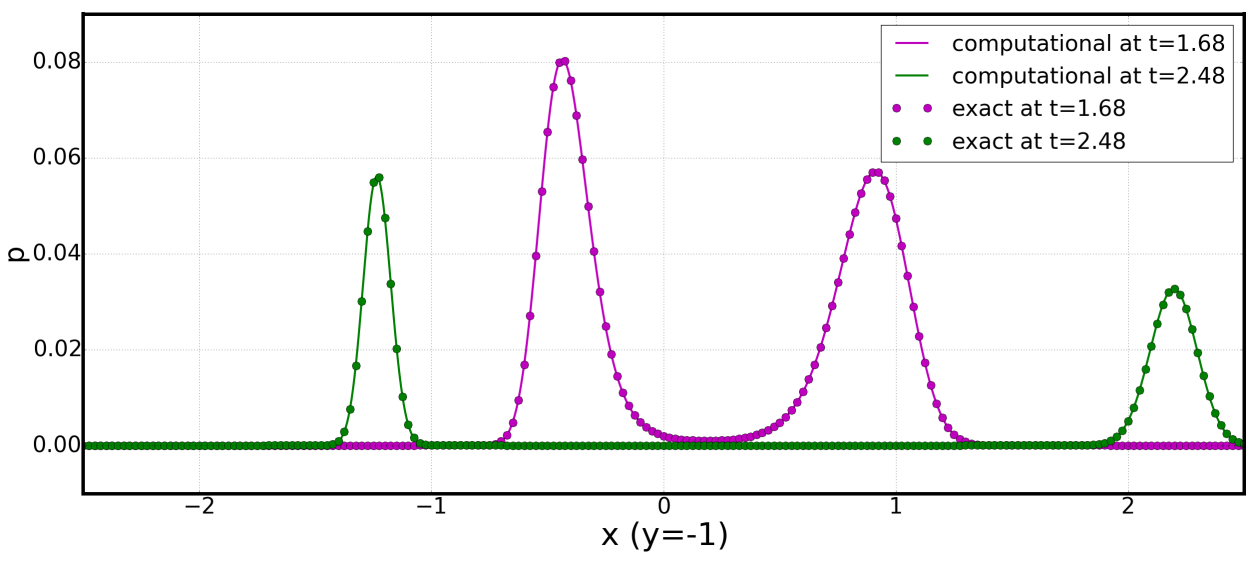

Figure 3. Value of $p$ along field line $y=-1$ at two instances as shown.

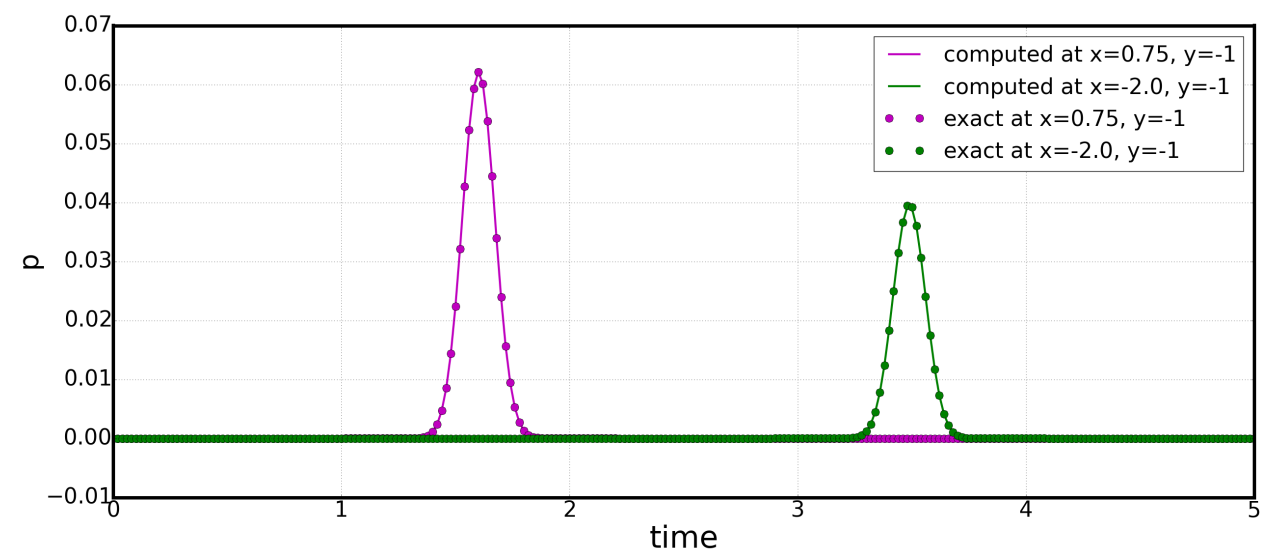

Figure 4. Values of $p$ as functions of time at two locations as shown.

\section{Assessment of acoustic shielding by a thin barrier}

In this section, we present an application of TD-FAST to the problem of acoustic shielding by a thin barrier. Recent NASA wind tunnel tests ${ }^{18}$ provide motivation for the selection of this case. While the ultimate goal is to couple the TD-FAST with time dependent CFD simulations and to predict the scattered field from the F3A31 Historical Baseline blade set in the 9x15 tunnel, initial predictions using a point source may be useful in setting up the problem geometry and illustrating the effect of source model on the scattered field. Based on reports of measured data, results up to a shaft order (SO) of 100 have been presented. This would essentially set an upper (model scale) frequency limit of approximately $10 \mathrm{kHz}$. One of the advantages of the time domain formulation used in the current paper is that scattering and shielding at all frequencies within the range of numerical resolution can be found at once in one single time domain computation. We will assess the accuracy of our numerical solution by comparing with an analytical solution.

Figure 5 shows the experimental setup and a geometrical modeling of the open rotor, the barrier, 
and microphone locations. For our computation, the noise source is modeled by a broadband point source, as given by $(14)$, located at $(x, y, z)=(0.84,0,0)$. The point source is introduced in the scattering computation through the source surface as described in the previous section. The source surface is formed by a rectangular box of dimension $0.8 \times 0.4 \times 0.4$ and discretized by 1400 elements, with 30, 10 and 10 elements in $\mathrm{x}, \mathrm{y}$ and $\mathrm{z}$ directions, respectively. In this example, the unit for spatial coordinates is meters.

The surface of the barrier facing the source region is located at $z=-0.625$ and the thickness of the barrier is 0.07. In the AFT configuration, the barrier extends from $x=0.84$ to $x=4.04$ in the $x$ direction. For the experimental results, ${ }^{18}$ the wind tunnel floor and ceiling are treated surfaces. However, the floor and ceiling are not included in the current predictions. Therefore, to reduce the effect of diffracted sound from the top and bottom edges of the barrier, the barrier in the computation is extended in the vertical $y$ direction to be -6 and 6 for the lower and upper edges, respectively (the experimental barrier extends from $-1.37<y<1.37$ ). A total of 91,022 elements are used with constant basis functions.

An observer line is included in the computation that coincides with the microphone traverse track in the experimental setup. The coordinates for the observer line are $(x, 0,-1.524),-4.5 \leq x \leq 3.0$. Field values on the observer line are computed using the integral relation (8) when solutions on the scattering surface are found.
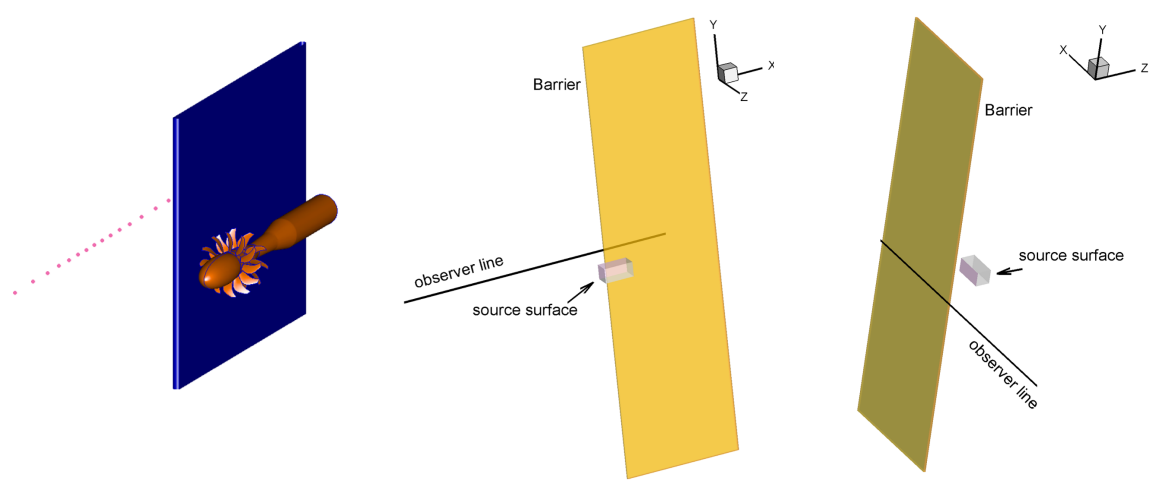

Figure 5. Schematics for the setup of barrier, source surface and an observer line.

In Figure 6, snapshots of the time domain solution are shown. To help visualize the solution by the time domain integral equation, values of pressure on a field plane, at $y=0$, are also computed and shown, in addition to the field line (drawn as the black line) that represents the microphone traverse track.

Solution time histories obtained with TD-FAST at three selected points are shown in Figure 7. The signals diffracted by the leading edge, trailing edge, and the top and bottom edges of the barrier are easily recognized and clearly separated as shown.

A distinct advantage of the time domain approach is that the frequency domain solutions at all frequencies within the time stepping resolution can be found through Fourier transform. Examples of contours of pressure at four selected frequencies are shown in Figure 8.

To help assess the accuracy of the numerical solution by TD-FAST, we compare the numerical solution with a known analytical solution of point source diffraction by a thin barrier. Spherical wave diffraction by a rigid half plane has been investigated previously ${ }^{14}$ and the solution, as well as other commonly used formulae for predicting sound diffraction by a thin barrier, reviewed. ${ }^{1,13}$ For completeness, the analytical solution and its numerical evaluation are discussed further in 


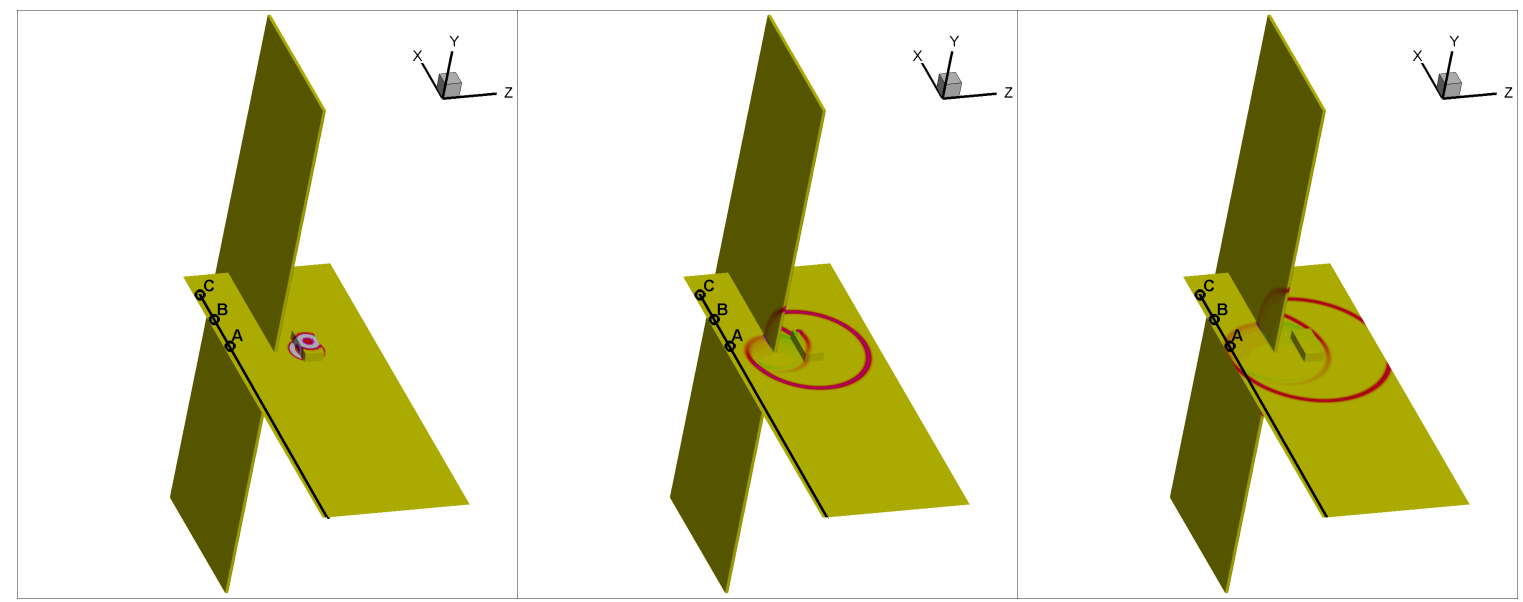

Figure 6. Instantaneous contours of pressure, showing the barrier, source surface and the observer line.

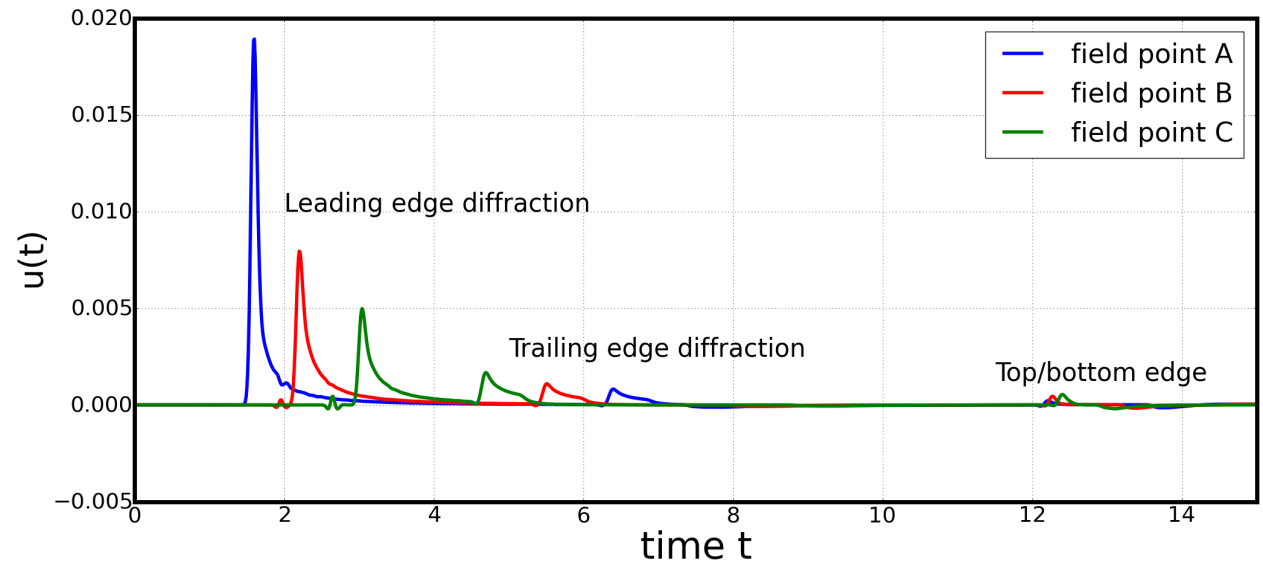

Figure 7. Time history of pressure at three locations on the observer line, A, B and C, as noted in Figure 6.

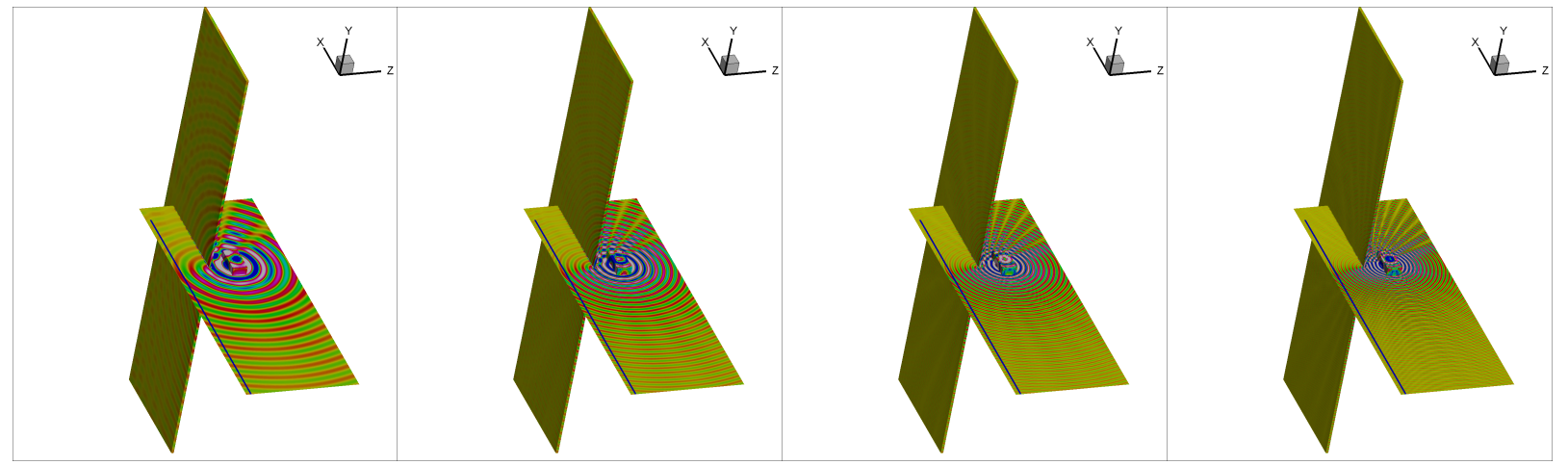

Figure 8. Frequency domain solutions converted from the time domain simulation, at $\omega=5 \pi, 10 \pi$, $15 \pi$, and $20 \pi$, from left to right. 
the Appendix. Figure 9 shows the comparison of the numerical and analytical solutions at six frequencies, $\omega=\pi, 2 \pi, 5 \pi, 10 \pi, 15 \pi$ and $20 \pi$. For the analytical solution, diffraction effects from both the leading and trailing edges are included. The agreements are in general very good. The minor discrepancies, which are seen increasing at higher frequencies, could be due to the finite thickness of the barrier.
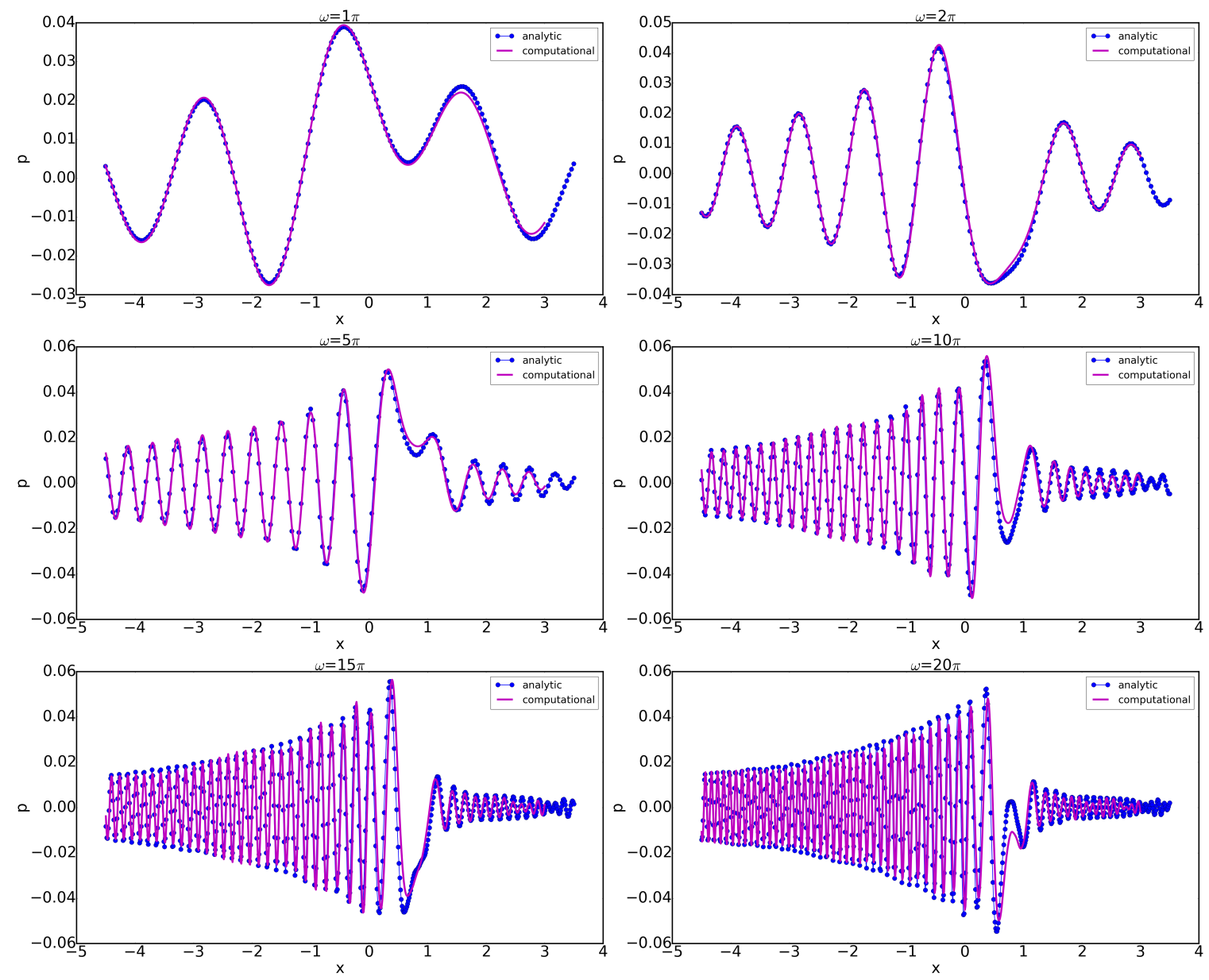

Figure 9. Comparison with the analytical solution at the frequencies as shown. 


\section{Assessment of spatial resolution of the boundary element method}

A detailed analysis on the accuracy and resolution of the temporal basis function $\Psi\left(t-t_{n}\right)$ used for the solution of the time domain boundary integral equation has been carried out. ${ }^{11}$ While the most commonly used temporal basis functions are usually formulated using the Lagrange interpolation polynomials, it was shown previously ${ }^{11}$ that the coefficients for the basis polynomials can be optimized, in exchange for a lowered formal order of accuracy, to extend the temporal resolution in the frequency space. In this section, we present a study on the spatial resolution of the boundary element method with respect to the spatial basis function $\phi_{i}\left(\boldsymbol{r}_{s}\right)$ in (15).

We consider the scattering and shielding of an acoustic point source by a flat plat, as shown in Figure 10. The plate has a dimension of $[-0.5,0.5] \times[-0.5,0.5] \times[-0.1,0.1]$. The point source is located at $(x, y, z)=(0,0,1)$. To study the spatial resolution of the boundary element method, a series of computations are carried out where the number of elements used in the computation is increased. For convenience of discussion, the top and bottom surfaces of the plate, at $z=-0.1$ and 0.1 , respectively, are discretized by $N_{x} N_{y}$ elements, where $N_{x}$ and $N_{y}$ are the number of elements in the $x$ and $y$ directions, respectively. The number of elements in the $z$ direction will be denoted by $N_{z}$.

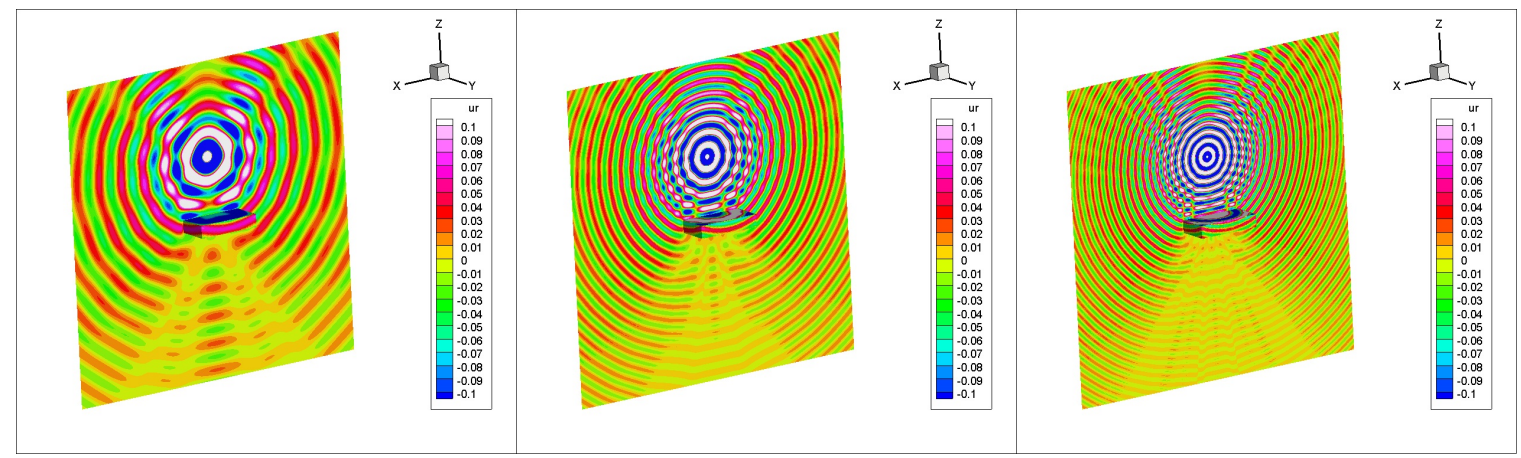

Figure 10. Frequency domain solutions of scattering by a flat plate, converted from the time domain solution at $\omega=5 \pi, 10 \pi$ and $15 \pi$, from left to right.

Figure 10 shows contour plots of the frequency domain solution converted from the time domain simulations, computed using $N_{x}=N_{y}=80$ and $N_{z}=16$. The frequency domain solutions along a field line of coordinates $(x, 0,-2.5),-2.5 \leq x \leq 2.5$, are shown in Figure 11 as the number of elements used in the computation increases from $20 \times 20 \times 4$ to $80 \times 80 \times 16$.
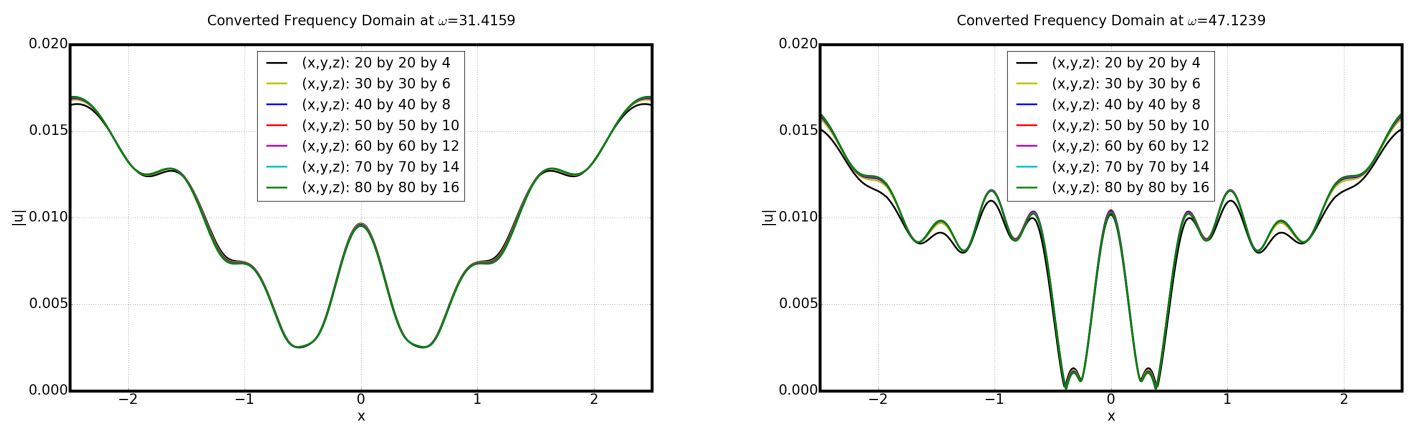

Figure 11. Frequency domain solution along $y=-1$. The number of elements used are as noted. 
Using the solution computed by $80 \times 80 \times 16$ as the reference solution, the relative errors in L2 norms are plotted in Figure 12 as a function of points per wavelength used in the computation. Here two metrics are used for the evaluation of spatial resolution, namely, points-per-wavelength (PPW) and points-per-wavelength-squared $\left(\mathrm{PPW}^{2}\right)$, which are defined as

$$
\begin{gathered}
P P W=\frac{2 \pi n_{e} N_{x}}{k L_{x}} \\
P P W^{2}=\frac{4 \pi^{2} \times(\text { total degrees of freedom })}{k^{2} \times(\text { surface area })}=\frac{4 \pi^{2} n_{e}\left[2 N_{x} N_{y}+2\left(N_{x}+N_{y}\right) N_{z}\right]}{k^{2}\left[2 L_{x} L_{y}+2\left(L_{x}+L_{y}\right) L_{z}\right]}
\end{gathered}
$$

where $n_{e}$ is the number of nodes per element and $k=\omega / c$ is the wavenumber, and $L_{x}, L_{y}$ and $L_{z}$ are the length of the sides of the plate in $x, y$ and $z$ directions, respectively. The points-per-wavelength metric measures the resolution along one direction on the surface, while the points-per-wavelengthsquared measures the resolution over the entire surface.

Results in Figure 12 show that, quite surprisingly, the relative error measured in L2 norm becomes as small as $2 \%$ when PPW is only 5 , or when $\mathrm{PPW}^{2}$ is only 25 , with only constant basis functions $\left(n_{e}=1\right)$ being used. We should also point out that, although the basis functions used are of zeroth order, the integrations over each element are computed by high-order Gauss quadrature on a 6x6 grid. ${ }^{10}$
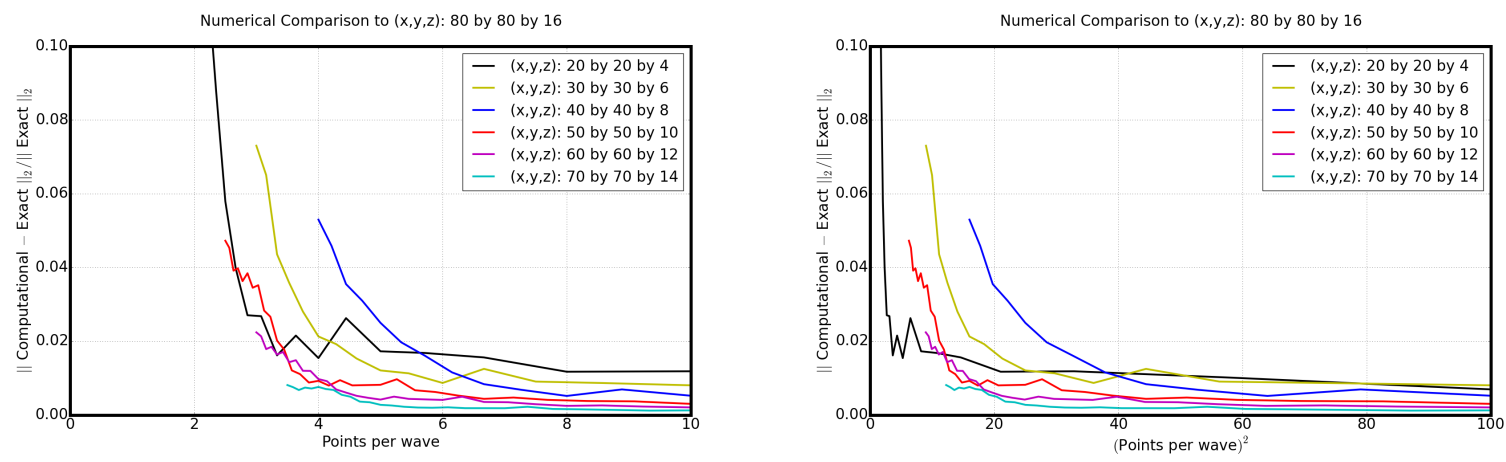

Figure 12. Left: relative error vs. Points-per-wavelength; Right: relative error vs. Points-perwavelength-squared

For another case, relative errors of the scattering by a sphere are shown in Figure 13. The sphere is centered at $(x, y, z)=(0,0,0)$ with a radius of 0.5 , and a point source is located at $(x, y, z)=(0,0,1)$. Constant elements are used. By comparing the computational solution along a field line $(x, 0,-2.5)$, $-2.5 \leq x \leq 2.5$, with the exact solution, the relative error vs. the points-per-wavelength-squared is plotted. Again, a similar trend is observed, as the relative error is reduced to less than $2 \%$ when $\mathrm{PPW}^{2}$ is 25 or more. It appears that the use of constant elements keeps the overall problem size small while the high-order integration helps maintain accuracy. Further investigation of the spatial resolution of boundary element method is to be conducted in future studies.

\section{Conclusions}

In this paper, a formulation for the time domain boundary integral equation has been extended to cases where the constant mean flow can be in an arbitrary direction. In addition, the current formulation is written in a way that a source surface can be naturally included. Introduction 
Numerical Comparison to Sphere Exact Solution

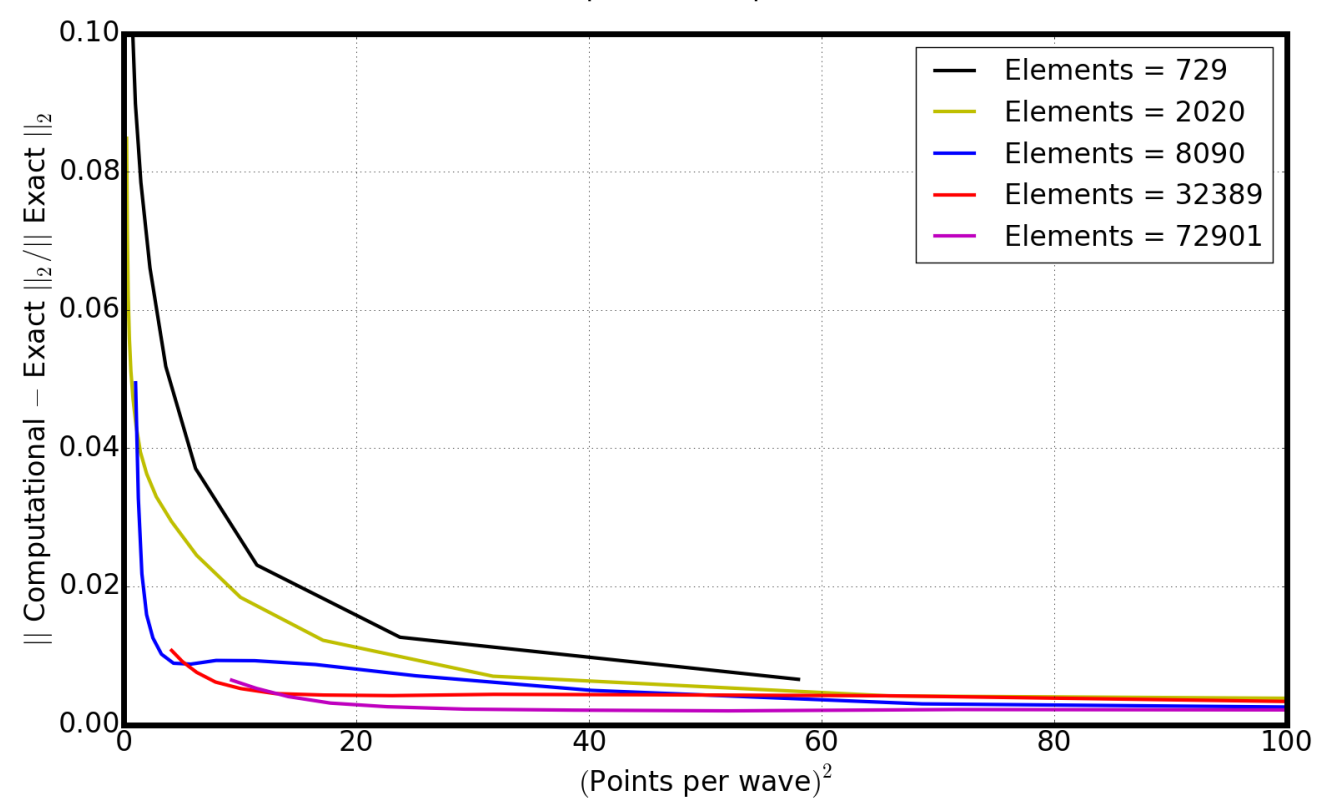

Figure 13. Relative L2 norm of errors for scattering by a sphere.

of source surfaces greatly increases the flexibility of TD-FAST and creates a convenient interface for coupling with CFD simulations. The accuracy of the new formulation has been assessed by comparing the computed and analytical solutions for fundamental configurations with very good agreement. Furthermore, spatial resolution of the time domain boundary element method has been assessed using points per wavelength metrics. It is found that, using only constant basis function and high-order quadrature for surface integration, relative errors measured by the L2 norm can be less than $2 \%$ when the surface spatial resolution is 5 points-per-wavelength (PPW) or 25 pointsper-wavelength-squared $\left(\mathrm{PPW}^{2}\right)$.

\section{Acknowledgements}

F. Q. Hu and M. E. Pizzo are supported by a NASA Cooperative Agreement, NNX11AI63A. M. E. Pizzo is also supported in part by an Old Dominion University Modeling and Simulation graduate fellowship. This work used the Extreme Science and Engineering Discovery Environment (XSEDE), which is supported by National Science Foundation grant number OCI-1053575.

\section{References}

${ }^{1}$ J. J. Bowman, T. B. A. Senior and P. L. E. Uslenghi (eds), Electromagnetic and Acoustic Scattering by Simple Shapes, North-Holland, Amsterdam, 1969.

${ }^{2}$ Y. Brick and A. Boag, "Multilevel nonuniform grid algorithm for acceleration of integral equation-based solvers for acoustic scattering", IEEE transaction on ultrasonic, ferroelectrics, and frequency control, Vol. 57, 261-273, 2010.

${ }^{3}$ O. M. Bucci, and G. Franceschetti, On the spatial bandwidth of scattered fields, IEEE Trans. Antennas Propag., Vol. 35, 1445-1455, 1987.

${ }^{4}$ A. J. Burton and G. F. Miller, The application of integral equation methods to the numerical solution of some exterior boundary-value problems, Proc.R.Soc.London, Ser A, Vol. 323, 201-210, 1971. 
${ }^{5}$ D. J. Chappell, P. J. Harris, D. Henwood and R. Chakrabarti, "A stable boundary element method for modeling transient acoustic radiation", Journal of Acoustical Society of America, Vol. 120, No. 1, 74-80, 2006.

${ }^{6}$ Dowling, A. P. and Ffowcs Williams, J. E., Sound and Sources of Sound, Horwood Publishing, Westergate, 1983.

${ }^{7}$ A. A. Ergin, B. Shanker and E. Michielssen, "Analysis of transient wave scattering from rigid bodies using a Burton-Miller approach", Journal of Acoustical Society of America, Vol. 106, No. 5, 2396-2404, 1999.

${ }^{8}$ C. L. Epstein, L. Greengard and T. Hagstrom, "On the stability of time-domain integral equations for acoustic wave propagation", arXiv preprint, arXiv:1504.04047, 2015.

${ }^{9}$ Y. Guo, Computation of sound propagation by boundary element method, NASA Contract Report, NAS1-00086A003, 2005.

${ }^{10} \mathrm{~F} . \mathrm{Q} . \mathrm{Hu}$, An efficient solution of time domain boundary integral equations for acoustic scattering and its acceleration by Graphics Processing Units, AIAA paper 2013-3018, 2013.

${ }^{11}$ F. Q. Hu, Further Development of a Time Domain Boundary Integral Equation Method for Aeroacoustic Scattering Computation, AIAA paper 2014-3194, 2014.

${ }^{12}$ A. D. Jones and F. Q. Hu, A three-dimensional time-domain boundary element method for the computation of exact Green's functions in acoustic analogy , AIAA paper 2007-3479, 2007

${ }^{13} \mathrm{Li}, \mathrm{K} . \mathrm{M}$. and Wong, H. Y., "A review of commonly used analytical and empirical formulae for predicting sound diffracted by a thin screen," Applied Acoustics, Vol. 66, 45-76, 2005.

${ }^{14}$ H. M. MacDonald, "A class of diffraction problems", Proc. London Math. Soc., Vol. 14, 410-427, 1915.

${ }^{15}$ J. Meng, A. Boag, V. Lomakin and E. Michielssen, "A multi-level Cartesian non-uniform grid time domain algorithm", Journal of Computational Physics, Vol. 229, 8430-8444, 2010.

${ }^{16} \mathrm{~L}$. Morino, Is there a difference between aeroacoustics and aerodynamics? An aeroelastician's view point, AIAA Journal, Vol. 41, 1209-1223, 2003.

${ }^{17}$ P. M. Morse and K. U. Ingard, Theoretical Acoustics, Princeton, 1986.

${ }^{18}$ D. Stephens and E. Envia, Acoustic Shielding for a Model Scale Counter-rotation open rotors, AIAA 2011-2940, 2011.

${ }^{19}$ Tinetti, A. F., Dunn, M. H., and Pope, D. S., "Fast Scattering Code (FSC) Users Manual, Version 2.0," NASA/CR2006-214510, October 2006.

${ }^{20}$ S. F. Wu, Nonuniqueness of solutions to extended Kirchhoff integral formulations, Journal of Acoustical Society of America, Vol. 93, 683-695, 1993.

${ }^{21} \mathrm{P}$. Zhang and T. W. Wu, A hypersingular integral formulation for acoustic radiation in moving flows, Journal of Sound and Vibration, Vol. 206, 309-326, 1997.

\section{Appendix}

For completeness, the analytical solution for spherical sound diffraction by a semi-infinite plate is given below.

The diffraction problem has been solved under the assumption of zero mean flow ${ }^{14}$ and further details have been provided in various references. ${ }^{1,13}$ Let a coordinate system $(X, Y, Z)$ be introduced with the origin set on a point at the leading edge, the $Z$ coordinate along the edge of the plate and the $X$ coordinate in the direction of the plate perpendicular to $Z$, as shown in Figure 14 . The $Y$ coordinate is in the direction opposite of the source point.

Let the cylindrical coordinates of the source and observer points be $\left(r_{s}, \theta_{s}, z_{s}\right)$ and $\left(r_{o}, \theta_{o}, z_{o}\right)$, respectively. Define

$$
\begin{aligned}
& R_{1}=\sqrt{r_{s}^{2}+r_{o}^{2}-2 r_{s} r_{o} \cos \left(\theta_{s}-\theta_{o}\right)+\left(z_{s}-z_{o}\right)^{2}} \\
& R_{2}=\sqrt{r_{s}^{2}+r_{o}^{2}-2 r_{s} r_{o} \cos \left(\theta_{s}+\theta_{o}\right)+\left(z_{s}-z_{o}\right)^{2}}
\end{aligned}
$$

and

$$
R^{\prime}=\sqrt{\left(r_{s}+r_{o}\right)^{2}+\left(z_{s}-z_{o}\right)^{2}}
$$

15 of 17 


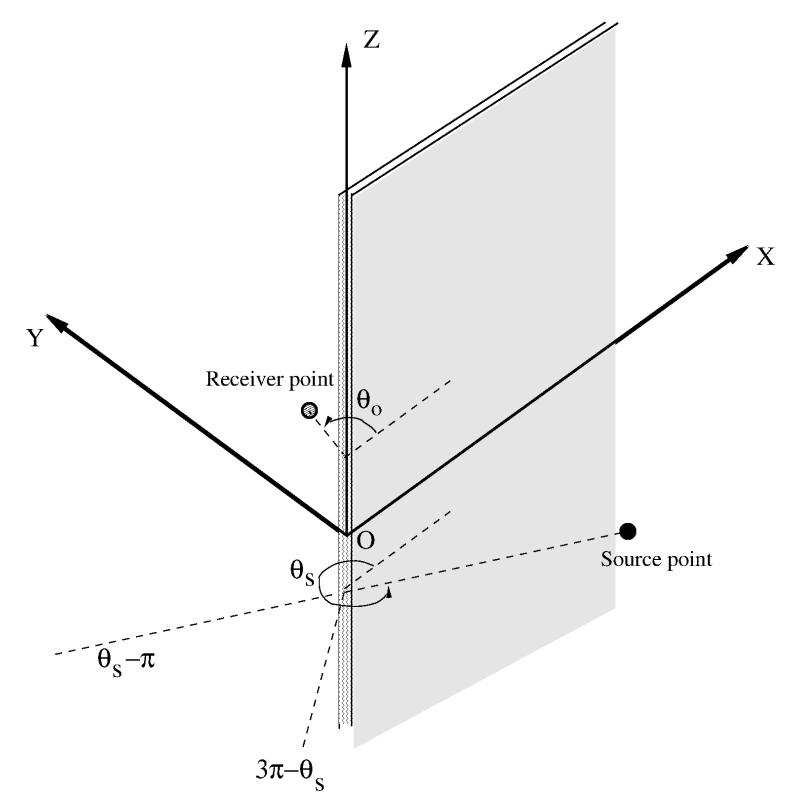

Figure 14. A schematic diagram showing the coordinates of the source and observer points. For the analytical solution, the plate is assumed to be infinitely thin.

Further, let $K$ be the wave number and, following Li et al. ${ }^{13}$ define respectively the direct, reflected and diffracted sound as

$$
\begin{gathered}
p_{d}(X, Y, Z, K)=\frac{e^{i K R}}{4 \pi R_{1}} \\
p_{r}(X, Y, Z, K)=\frac{e^{i K R_{2}}}{4 \pi R_{2}} \\
p_{D}(X, Y, Z, K)=\frac{i K \operatorname{sign}\left(\zeta_{1}\right)}{4 \pi} \int_{\left|\zeta_{1}\right|}^{\infty} \frac{H_{1}^{(1)}\left(K R_{1}+\mu^{2}\right)}{\sqrt{\mu^{2}+2 K R_{1}}} d \mu+\frac{i K \operatorname{sign}\left(\zeta_{2}\right)}{4 \pi} \int_{\left|\zeta_{2}\right|}^{\infty} \frac{H_{1}^{(1)}\left(K R_{2}+\mu^{2}\right)}{\sqrt{\mu^{2}+2 K R_{2}}} d \mu
\end{gathered}
$$

where

$$
\zeta_{1}=\operatorname{sign}\left(\left|\theta_{s}-\theta_{o}\right|-\pi\right) \sqrt{K\left(R^{\prime}-R_{1}\right)}, \quad \zeta_{2}=\operatorname{sign}\left(\theta_{s}+\theta_{o}-\pi\right) \sqrt{K\left(R^{\prime}-R_{2}\right)}
$$

Then, the sound pressure at the observer point point $\left(r_{o}, \theta_{o}, z_{o}\right)$ is:

If $\theta_{s}<\theta_{o} \leq 2 \pi$ :

$$
p_{o}(X, Y, Z, K)=p_{d}+p_{r}+p_{D}
$$

If $\theta_{s}-\pi<\theta_{o}<3 \pi-\theta_{s}$ :

$$
p_{o}(X, Y, Z, K)=p_{d}+p_{D}
$$

If $\theta_{o}<\theta_{s}-\pi$ : 


$$
p_{o}(X, Y, Z, K)=p_{D}
$$

The integrals can be evaluated by numerical quadrature and asymptotic expansion of the Hankel function.

In the presence of a mean flow in the direction of the $x$ axis, $\boldsymbol{M}=\left(M_{1}, 0,0\right)$, the solution in the physical coordinates of $(x, y, z)$ at a wave number $k$ can be found by making use of the PrandtlGlauert transformation:

$$
(X, Y, Z)=\left(\frac{1}{\sqrt{1-M_{1}^{2}}} x, y, z\right), T=\sqrt{1-M_{1}^{2}} t+\frac{M_{1}}{c \sqrt{1-M_{1}^{2}}} x
$$

Then, the frequency domain solution with the mean flow effect can be found to be

$$
p(x, y, z, k)=\frac{1}{\sqrt{1-M_{1}^{2}}} p_{o}(X, Y, Z, K) e^{-i k M_{1}\left(x-x_{s}\right) /\left(1-M_{1}^{2}\right)}
$$

where $x_{s}$ is the $x$ coordinate of the source point and $p_{o}(X, Y, Z, K)$ is that computed by (18)-(20) with

$$
K=\frac{k}{\sqrt{1-M_{1}^{2}}}
$$

